\title{
Quantum dissipative dynamics of a bistable system in the sub-Ohmic to super-Ohmic regime
}

\section{Luca Magazzù ${ }^{1,2}$, Angelo Carolloํㅜ, Bernardo Spagnolo ${ }^{1,2,3}$ and Davide Valenti ${ }^{1}$}

${ }^{1}$ Dipartimento di Fisica e Chimica, Group of Interdisciplinary Theoretical Physics, Università di Palermo and CNISM, Unità di Palermo, Viale delle Scienze, Edificio 18, I-90128 Palermo, Italy

${ }^{2}$ Radiophysics Department, Lobachevsky State University of Nizhny Novgorod, Russia

${ }^{3}$ Istituto Nazionale di Fisica Nucleare, Sezione di Catania, Italy

E-mail: luca.magazzu@unipa.it, angelo.carollo@unipa.it, bernardo.spagnolo@unipa.it and davide.valenti@unipa.it

Received 19 October 2015

Accepted for publication 4 January 2016

Published 20 May 2016

Online at stacks.iop.org/JSTAT/2016/054016

doi:10.1088/1742-5468/2016/05/054016

\begin{abstract}
We investigate the quantum dynamics of a multilevel bistable system coupled to a bosonic heat bath beyond the perturbative regime. We consider different spectral densities of the bath, in the transition from subOhmic to super-Ohmic dissipation, and different cutoff frequencies. The study is carried out by using the real-time path integral approach of the FeynmanVernon influence functional. We find that, in the crossover dynamical regime characterized by damped intrawell oscillations and incoherent tunneling, the short time behavior and the time scales of the relaxation starting from a nonequilibrium initial condition depend nontrivially on the spectral properties of the heat bath.
\end{abstract}

Keywords: dissipative systems (theory), quantum transport in one-dimension, quantum transport 


\section{Contents}

1. Introduction 2

2. Model 3

3. Feynman-Vernon influence functional approach $\quad 6$

3.1. Exact path integral expression for the reduced density matrix . . . . . . 6

3.2. Generalized master equation within the generalized noninteracting blip approximation ...................... 7

4. Dynamics and relaxation times 11

$\begin{array}{ll}\text { 5. Conclusions } & 16\end{array}$

$\begin{array}{ll}\text { Acknowledgments } & 16\end{array}$

Appendix. Numerical scheme for the generalized master equation 16

$\begin{array}{ll}\text { References } & 18\end{array}$

\section{Introduction}

Real quantum systems are always in contact with noisy environments causing dissipation and decoherence. Quantum dissipation by phononic or electromagnetic environments is described well by the celebrated Caldeira-Leggett model [1] in which a quantum particle, the open system, is linearly coupled to a reservoir of $N$ independent quantum harmonic oscillators. Even if the coupling with the individual oscillators is weak, the overall dissipative effect may be strong, especially for macroscopic systems such as superconducting quantum interference devices $[2,3]$. In the thermodynamical limit $N \rightarrow \infty$ the reservoir is a bosonic heat bath and is described by the spectral density function $J(\omega)$, usually assumed to be of the form $\omega^{s}$ with a high-frequency cut-off. The special case $s=1$ gives the so-called Ohmic dissipation. In this case the quantum Langevin equation for the position operator of the particle has a memoryless damping kernel (frequency independent friction) and, in the classical limit $\hbar \rightarrow 0$, the heat bath reduces to a classical white noise source [3].

In this work we study the bistable dynamics of a quantum particle coupled to an environment of which we vary the spectral density. Bistable potentials are ubiquitous both in the classical and quantum context [4] as they are used to study the passage between potential minima separated by an energy barriers that can be classically surmounted or crossed via quantum tunneling. The quantum regime is generally characterized by the presence of several energy levels below the potential barrier. Due to the strong nonlinearity of the potential, these levels are organized in tunneling doublets with internal energy separation much smaller than the interdoublet separation. As a consequence the tunneling dynamics occurs on time scales much larger than that of the oscillations internal to the well, whose frequency is given by the separation between the first and the second doublet. 
The usual way of describing the tunneling dynamics in the presence of dissipation is by means of the two-level system (TLS) approximation. Within this approximation the Hilbert space of the particle is truncated to that spanned by the first two energy eigenstates. Despite its simplicity, the spin-boson model, resulting from the TLS truncation of the Caldeira-Leggett model, displays several nontrivial features such as the coherent-incoherent transition, as the coupling strength is increased, and a further transition to the localized phase at stronger coupling, the occurrence of which depends on the spectral content of the environment $[3,5,6]$.

However, when the temperature is of the same order of magnitude of the separation between the first and the second energy doublet, the TLS approximation is questionable, as higher energy levels are non-negligibly involved in the dynamics, and a beyondTLS description becomes then necessary [7-10]. Nevertheless, theoretical investigations have been mainly focused on the dissipative dynamics of the spin-boson model [11-17].

Regardless of the assumptions on the open system's Hilbert space, the interaction with an environment beyond the weak coupling regime yields intricate time-nonlocal effects in the reduced dynamics. To deal with these intricacies, several numerical techniques based on different strategies have been developed so far. These include the quasi-adiabatic propagator path integrals (QUAPI) [18], variationally optimized master equation approach [19], numerical renormalization group techniques [20-22], Monte Carlo path integral evaluation [23], methods based on the wave function propagation [24], stochastic techniques [25], hierarchy equations [26] and hybrid stochastic hierarchy equations approaches [27].

In this work, we consider the dynamics of a dissipative bistable system, beyond the TLS approximation, in a temperature regime in which the presence of the second energy doublet cannot be neglected. By using a nonperturbative generalized master equation with approximated kernels, derived within the Feynman-Vernon influence functional approach [28-30], we investigate a regime of dissipation which is outside of the validity of the perturbative Born-Markov master equation approach. We study the reduced dynamics by varying the exponent $s$ in the crossover from the sub-Ohmic $(s<1)$ to the super-Ohmic $(s>1)$ dissipation regime. We consider also the effects of changing the cutoff frequency $\omega_{c}$, i.e. the contribution of the high-frequency modes to the open dynamics. Recently, for a quantum dot modeled as a TLS interacting with bosonic and electronic environments at zero temperature, a study on the effects of varying the spectral density function from sub-Ohmic to super-Ohmic has been performed [31].

\section{Model}

The open system $S$ is a quantum particle of mass $M$ and position operator $\hat{q}$ in the presence of a double well potential $V$. According to the Caldeira-Leggett model, the particle interacts linearly with a heat bath $B$ of $N$ independent quantum harmonic oscillators of positions $\hat{x}_{j}$ and momenta $\hat{p}_{j}$. The full Hamiltonian of the model is

$$
\hat{H}=\hat{H}_{\mathrm{S}}+\hat{H}_{\mathrm{B}}+\hat{H}_{\mathrm{SB}}
$$


where $\hat{H}_{\mathrm{S}}=\hat{p}^{2} / 2 M+V(\hat{q})$ is the open system Hamiltonian and

$$
\hat{H}_{\mathrm{B}}+\hat{H}_{\mathrm{SB}}=\frac{1}{2} \sum_{j=1}^{N}\left[\frac{\hat{p}_{j}^{2}}{m_{j}}+m_{j} \omega_{j}^{2}\left(\hat{x}_{j}-\frac{c_{j}}{m_{j} \omega_{j}^{2}} \hat{q}\right)^{2}\right]
$$

is the free bath and system-bath (bilinear) interaction, whose strength is quantified by the set of couplings $c_{j}$. The renormalization term quadratic in $\hat{q}$ is introduced to give a purely dissipative bath, i.e. a spatially homogeneous dissipation [3].

The potential $V$ considered in this work is the double well potential with a couple of energy doublets under the barrier shown in figure 1. Following the parametrization used in [30], $V$ is given by the quartic function

$$
V(\hat{q})=\frac{M^{2} \omega_{0}^{4}}{64 \Delta U} \hat{q}^{4}-\frac{M \omega_{0}^{2}}{4} \hat{q}^{2}-\epsilon \hat{q} .
$$

The choice of the parameters of $V$ used in the present work is the same as that in [32], where the multilevel dynamics in a sub-Ohmic environment with high frequency cutoff was investigated. Specifically we assume a static bias $\epsilon=0.02 \sqrt{M \hbar \omega_{0}^{3}}$ and a potential barrier (at zero bias) $\Delta U=1.4 \hbar \omega_{0}$. With our choice of parameters the first four energy levels are organized in two doublets separated by a frequency gap $\Omega \sim \omega_{0}$ with internal frequency separations $\Omega_{1}, \Omega_{2} \ll \omega_{0}$ (see figure 1 ).

This double-doublet structure describes well that of the flux qubits used in the experiment on quantum annealing performed in [10]. In general, superconducting devices with externally tunable parameters [33-35] can produce theoretically and experimentally a variety of potential profiles and initial conditions. For such devices, typical values of $\omega_{0}$ are of the order of the $\mathrm{GHz}[33,36,37]$. In particular, the superconducting quantum devices of [33] are the reference physical systems for our analysis. Moreover this kind of devices operate in a wide range of temperatures and coupling strengths [38-40]. The double-doublet system (DDS) is also used for modeling atomic Bose-Einstein condensates in double well potentials [41] or proton transfer reactions [7].

In the present work we assume the following algebraic spectral density function with exponential cutoff

$$
J(\omega)=M \gamma_{s}\left(\omega / \omega_{\mathrm{ph}}\right)^{s-1} \omega \mathrm{e}^{-\omega / \omega_{c}}
$$

The bath is sub-Ohmic for $0<s<1$, Ohmic for $s=1$ and super-Ohmic for $s>1$. The parameter $\gamma_{s}$, with dimension of frequency, is the system-bath coupling strength. The phonon frequency $\omega_{\mathrm{ph}}$ is a characteristic frequency of the reservoir, introduced so that $J$ has the dimension of a mass times a frequency squared also for $s \neq 1$. In what follows we set $\omega_{\text {ph }}=\omega_{0}$.

In figure 2 we show the spectral density functions $J(\omega)$ in the sub-Ohmic, Ohmic, and super-Ohmic regimes for two values of the cutoff frequency. There, the density of low frequency modes is the highest in the sub-Ohmic regime. On the other hand, the 


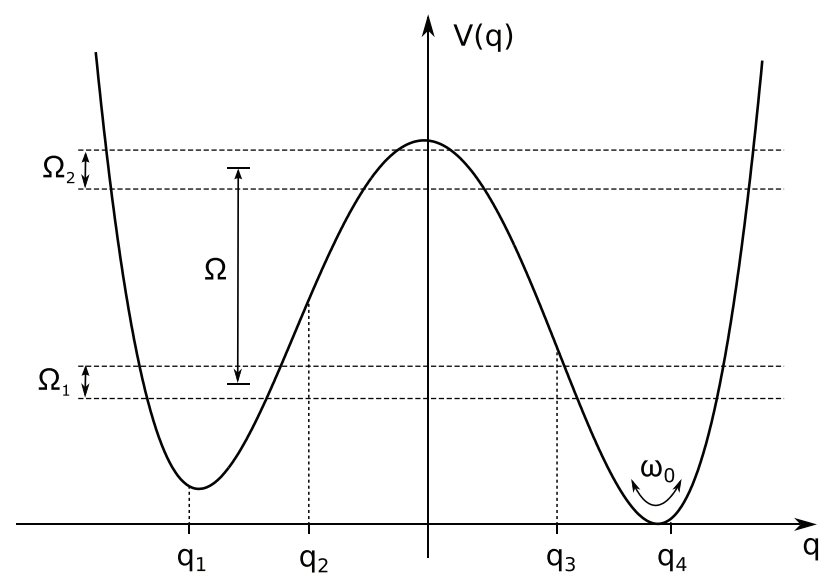

Figure 1. Potential $V_{0}$ with energy levels (horizontal lines) and DVR positions. The frequency $\omega_{0}$ is the oscillation frequency around the potential minima. The average inter-doublet frequency spacing, that is the characteristic frequency of the intrawell motion, is $\Omega=0.814 \omega_{0}$. The frequency spacings internal to the first and second energy doublet are $\Omega_{1}=0.123 \omega_{0}$ and $\Omega_{2}=0.149 \omega_{0}$, respectively. These two frequencies characterize the tunneling dynamics of the bistable system.

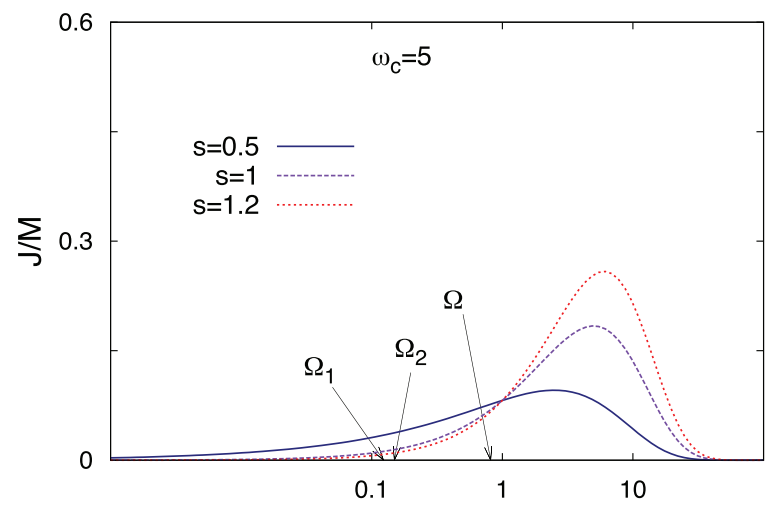

$\omega$

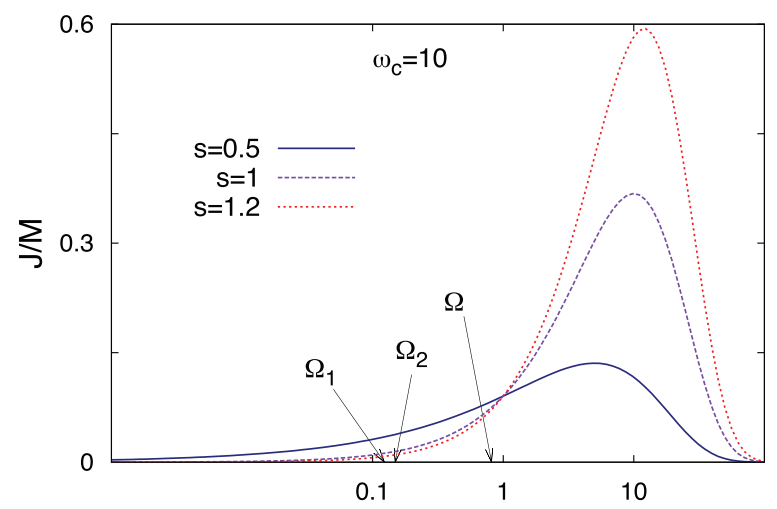

$\omega$

Figure 2. Bath spectral density function (see equation (4)) as a function of $\omega$ in $\log$ scale. Three values of $s$ are considered for each of the two values of the cutoff frequency $\omega_{c}=5$ (left panel) and $\omega_{c}=10$ (right panel). The characteristic frequencies of the isolated system, $\Omega$ and $\Omega_{i}$ (see figure 1 ), are displayed for reference. Frequencies are in units of $\omega_{0}$.

density of high frequency modes, especially at large cutoff frequency, is the largest in the super-Ohmic regime.

We assume that the environment has a physical cutoff at $\omega_{c}$, which may be the Debye frequency of the medium in which the system is immersed. The values of the cutoff frequency $\omega_{c}$ used here are taken in the range $5 \omega_{0}$ to $50 \omega_{0}$. The bath modes with frequencies up to the frequency scale set by $\omega_{0}$ affect the particle dynamics through the quantum friction modeled by the Caldeira-Leggett Hamiltonian (equation (1)) on the time scales of the intrawell motion or longer. The modes with frequencies $\omega \gtrsim \omega^{*}$, where $\omega_{0} \ll \omega^{*} \sim \omega_{c}$, affect the system dynamics by renormalizing the mass according to $M \rightarrow M+\Delta M_{\omega^{*}}$, with 


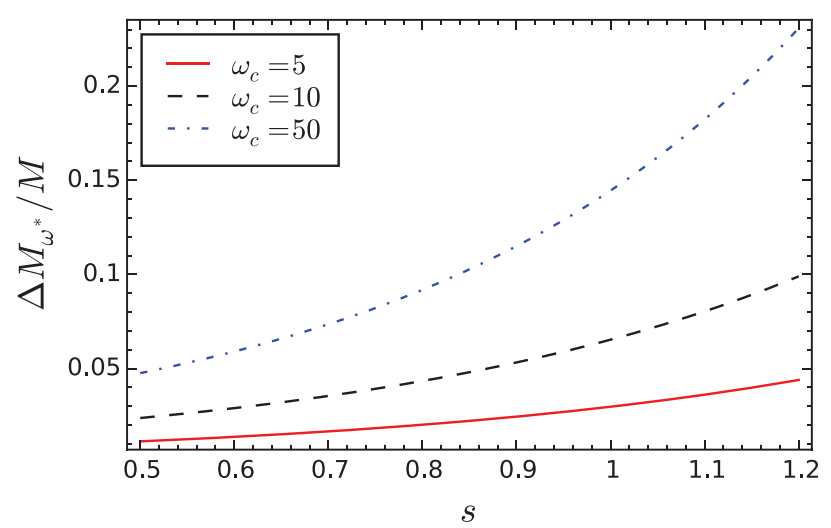

Figure 3. $\Delta M_{\omega^{*}} / M$ as a function of the exponent $s$ of the bath spectral density function (equation (5)), for different values of the cutoff frequency $\omega_{c}$. Parameters are: $\gamma_{s}=\pi / 2, \omega_{\mathrm{ph}}=1$, and $\omega^{*}=5$. Frequencies and $\gamma_{s}$ are in units of $\omega_{0}$.

$$
\begin{aligned}
\Delta M_{\omega^{*}} & =\frac{2}{\pi} \int_{\omega^{*}}^{\infty} \mathrm{d} \omega \frac{J(\omega)}{\omega^{3}} \\
& =\frac{2}{\pi} M \frac{\gamma_{s}}{\omega_{c}}\left(\frac{\omega_{c}}{\omega_{\mathrm{ph}}}\right)^{s-1} \Gamma\left(s-2, \omega^{*} / \omega_{c}\right),
\end{aligned}
$$

where $\Gamma(z, x)=\int_{x}^{\infty} \mathrm{e}^{-t} t^{z-1} \mathrm{~d} t$ is the incomplete gamma function, related to the Euler Gamma function by $\Gamma(z, 0)=\Gamma(z)$. This can be shown by considering the quantum Langevin equation for the system, as done in [3]. The magnitude of this mass renormalization due to the high frequency modes of the bath is shown in figure 3 as a function of the exponent $s$ of the spectral density function (see equation (4)). This behavior involves uniquely the features of the thermal bath. As a consequence it holds for a generic potential, and is not influenced by truncations of the Hilbert space of the particle or by specific structures of the energy levels, such as the double-doublet shown in figure 1. Moreover, while equation (5) is useful to get a physical picture of the effects on the particle's motion exerted by the various types of environment considered in this work, these effects are fully taken into account by our path integral approach for the reduced dynamics, with no explicit change in the mass.

\section{Feynman-Vernon influence functional approach}

\subsection{Exact path integral expression for the reduced density matrix}

The reduced dynamics of the open system $S$ is given by the trace of the full density matrix $\rho_{\mathrm{SB}}$ over the bath degrees of freedom

$$
\rho(t)=\operatorname{Tr}_{\mathrm{B}}\left[U\left(t, t_{0}\right) \rho_{\mathrm{SB}}\left(t_{0}\right) U^{\dagger}\left(t, t_{0}\right)\right] .
$$

The time evolution operator is $U\left(t, t_{0}\right)=\exp \left[-\mathrm{i} \hat{H}\left(t-t_{0}\right) / \hbar\right]$, with $\hat{H}$ the full Hamiltonian of the model given in equation (1). A factorized initial state of the type $\rho_{\mathrm{SB}}\left(t_{0}\right)=\rho\left(t_{0}\right) \otimes B^{\beta}$ 
is assumed, where $\rho\left(t_{0}\right)$ is an arbitrary state of $S$ and $B^{\beta}=\exp \left(-\beta \hat{H}_{\mathrm{B}}\right) / Z$ is the thermal state of the bath at inverse temperature $\beta=1 / k_{\mathrm{B}} T$.

The reduced density matrix at time $t$ in the position representation has matrix elements $\rho_{q q^{\prime}}=\left\langle q|\rho| q^{\prime}\right\rangle$ given by

$$
\rho_{q q^{\prime}}(t)=\int \mathrm{d} q_{0} \int \mathrm{d} q_{0}^{\prime} G\left(q, q^{\prime}, t ; q_{0}, q_{0}^{\prime}, t_{0}\right) \rho_{q_{0} q_{0}^{\prime}}\left(t_{0}\right),
$$

where the propagator $G$ is the double path integral over the paths of the left and right coordinates $q$ and $q^{\prime}$

$G\left(q, q^{\prime}, t ; q_{0}, q_{0}^{\prime}, t_{0}\right)=\int_{q_{0}}^{q} \mathcal{D} q(\tau) \int_{q_{0}^{\prime}}^{q^{\prime}} \mathcal{D}^{*} q^{\prime}(\tau) \mathrm{e}^{\frac{\mathrm{i}}{\hbar}\left(S[q(\tau)]-S\left[q^{\prime}(\tau)\right]\right)} \mathcal{F}_{\mathrm{FV}}\left[q(\tau), q^{\prime}(\tau)\right]$

The functional $S[q(t)]=\int_{t_{0}}^{t} \mathrm{~d} t^{\prime} \mathcal{L}_{S}\left(q\left(t^{\prime}\right), t^{\prime}\right)$ is the action functional for the bare system. The Feynman-Vernon $\mathcal{F}_{\mathrm{FV}}=\exp \left(-\Phi_{\mathrm{FV}}\right)$ derives from tracing over the bath degrees of freedom. The paths $q(\tau)$ and $q^{\prime}(\tau)$ of the left and right coordinates are coupled in a time nonlocal fashion in the influence phase functional $\Phi_{\mathrm{FV}}$, which, in terms of the combinations $x=q+q^{\prime}$ and $y=q-q^{\prime}$, takes on the following form

$$
\begin{aligned}
\Phi_{\mathrm{FV}}[y, x]= & \frac{1}{\hbar^{2}} \int_{t_{0}}^{t} \mathrm{~d} t^{\prime} \int_{t_{0}}^{t^{\prime}} \mathrm{d} t^{\prime \prime} y\left(t^{\prime}\right)\left[L^{\prime}\left(t^{\prime}-t^{\prime \prime}\right) y\left(t^{\prime \prime}\right)+\mathrm{i} L^{\prime \prime}\left(t^{\prime}-t^{\prime \prime}\right) x\left(t^{\prime \prime}\right)\right] \\
& +\mathrm{i} \frac{\lambda}{\hbar} \int_{t_{0}}^{t} \mathrm{~d} t^{\prime} x\left(t^{\prime}\right) y\left(t^{\prime}\right) .
\end{aligned}
$$

Here $L^{\prime}$ and $L^{\prime \prime}$ are the real and imaginary part of bath correlation function defined by

$$
L(t)=\frac{\hbar}{\pi} \int_{0}^{\infty} \mathrm{d} \omega J(\omega)\left[\operatorname{coth}\left(\frac{\hbar \omega \beta}{2}\right) \cos \omega t-\mathrm{i} \sin \omega t\right],
$$

and the constant $\lambda=\int_{0}^{\infty} \mathrm{d} \omega J(\omega) / \omega=M \gamma_{s} \Gamma(s) \omega_{c}\left(\omega_{c} / \omega_{\mathrm{ph}}\right)^{s-1}$ is proportional to the so-called reorganization energy, which measures the overall system-bath coupling [6].

\subsection{Generalized master equation within the generalized noninteracting blip approximation}

If the potential $V$ is harmonic, the propagator for the reduced density matrix can be evaluated analytically [42] yielding the exact dynamics of the dissipative harmonic oscillator. However, such an analytic solution does not exist for the nonlinear potential considered here. Nevertheless, an approximate treatment is possible in a temperature regime where the system is not going to be excited to high energy levels and the potential barrier is crossed by tunneling. In the present work this approximated treatment is based on the spatial discretization resulting from the truncation of the Hilbert space to that spanned by the first four energy eigenstates $\left|E_{1}\right\rangle, \ldots,\left|E_{4}\right\rangle$. Performing on this restricted basis the unitary transformation $\mathbf{T}$, which diagonalizes the position operator $\hat{q}$ of matrix elements $\left\langle E_{i}|\hat{q}| E_{j}\right\rangle$, we pass to the discrete variable representation (DVR) $[43,44]$. The DVR is given by the set of functions 


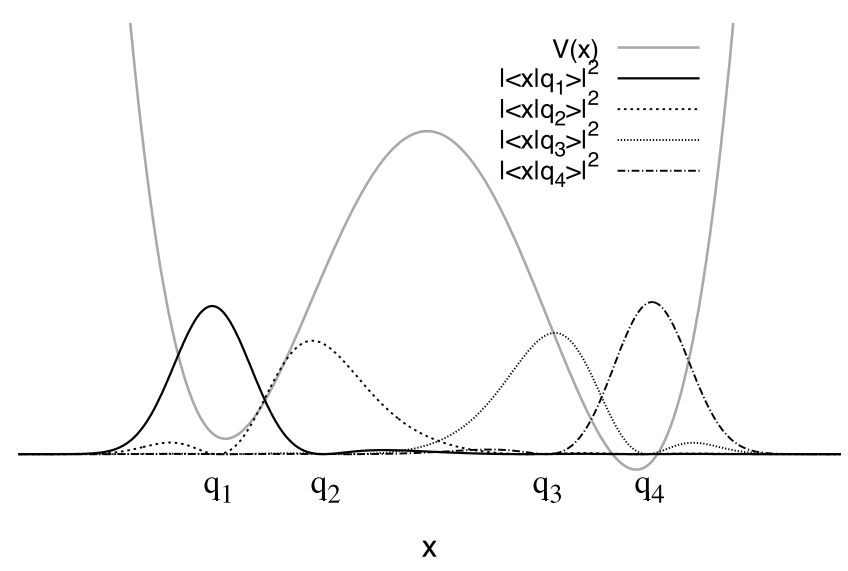

Figure 4. Potential profile (solid grey line) and probability densities of the four DVR states.

$$
\left|q_{j}\right\rangle=\sum_{k=1}^{4} T_{j k}^{*}\left|E_{k}\right\rangle
$$

focused around the four position eigenvalues $q_{1}, \ldots, q_{4}$ depicted in figure 1 . The diagonal element $\rho_{i i}=\left\langle q_{i}|\rho| q_{i}\right\rangle$ of the reduced density matrix in the DVR, i.e. the population of the state $\left|q_{i}\right\rangle$, is the probability of finding the particle in a region of space localized around $q_{i}$. In figure 4 we show the probability densities of the four DVR states for our specific problem.

In passing to the DVR, the exact reduced density matrix given in equation (7) assumes the form $\rho_{q q^{\prime}}(t)=\sum_{q_{0}, q_{0}^{\prime}} G\left(q, q^{\prime}, t ; q_{0}, q_{0}^{\prime}, t_{0}\right) \rho_{q_{0} q_{0}^{\prime}}\left(t_{0}\right)$, where the propagator has the formal expression

$$
G\left(q, q^{\prime}, t ; q_{0}, q_{0}^{\prime}, t_{0}\right)=\sum_{n=0}^{\infty} \int_{t_{0}}^{t} D_{n}\{t\} \mathcal{A}[q] \mathcal{A}^{*}\left[q^{\prime}\right] \exp \left(-\Phi[\xi, \chi]_{\mathrm{FV}}\right) .
$$

The sum is over the number $n$ of transitions of the paths and the symbol $\int_{t_{0}}^{t} D_{n}\left\{t_{j}\right\}$ denotes the sum $\sum_{\text {paths }_{n}} \int_{t_{0}}^{t} \mathrm{~d} t_{n} \int_{t_{0}}^{t_{n}} \mathrm{~d} t_{n-1} \ldots \int_{t_{0}}^{t_{2}} \mathrm{~d} t_{1}$ over all path configurations with $n$ transitions at times $t_{j}$. In equation (12), the amplitude $\mathcal{A}[q]$ for the path $q\left(t_{k}\right)$ of the isolated system includes the product of the transition amplitudes per unit time $\Delta_{i j}=\left\langle q_{i}\left|\hat{H}_{S}\right| q_{j}\right\rangle / \hbar$, relative to the transitions $\left|q_{i}\right\rangle \rightarrow\left|q_{j}\right\rangle$. The influence of the environment is encapsulated in the Feynman-Vernon influence phase, whose expression in the DVR is [45]

$$
\Phi[\xi, \chi]_{\mathrm{FV}}=-\sum_{i=1}^{n} \sum_{j=0}^{i-1}\left[\xi_{i} Q^{\prime}\left(t_{i}-t_{j}\right) \xi_{j}+\mathrm{i} \xi_{i} Q^{\prime \prime}\left(t_{i}-t_{j}\right) \chi_{j}\right],
$$

where the so-called charges at time $t_{k}$ are defined by $\xi_{k}=y_{k}-y_{k-1}$ and $\chi_{k}=x_{k}-x_{k-1}$. The pair interaction $Q(t)=Q^{\prime}(t)+\mathrm{i} Q^{\prime \prime}(t)$, which couples the $\xi$ - and $\chi$-charges, is related to the bath force correlation function, defined in equation (10), by $L(t)=\hbar^{-2} \mathrm{~d}^{2} Q(t) / \mathrm{d} t^{2}$. In terms of the parameter $\lambda \equiv \lambda_{s}$ introduced in equation (9), the pair interaction for a generic exponent $s$ reads [3] 


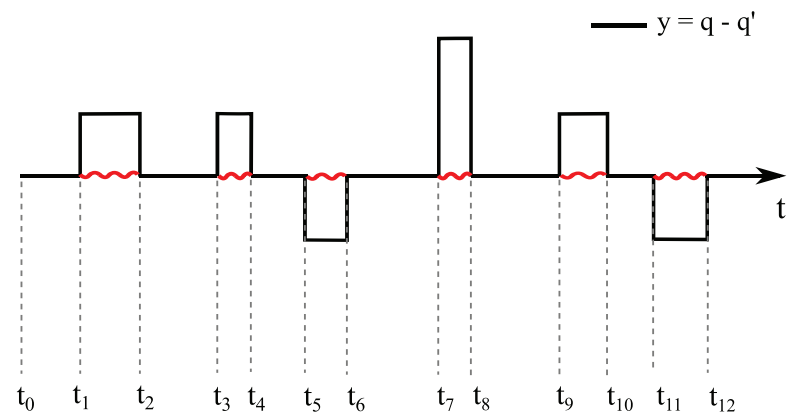

Figure 5. Example of a path of the reduced density matrix in the coordinate $y=q-q^{\prime}$. The path consists of five intrawell blips and one tunneling blip between $t_{7}$ and $t_{8}$. The intrablip interactions retained in the influence functional (see equation (13)) are denoted by the red wavy lines.

$Q(t)=\frac{\lambda_{s}}{\pi \hbar(s-1)}\left[1-\left(1+\mathrm{i} \omega_{c} t\right)^{1-s}+\kappa^{s-1} f_{\kappa}(s-1, t)\right]$

where $\kappa=k_{\mathrm{B}} T / \hbar \omega_{c}$ and $f_{\kappa}(s, t)=2 \zeta(s, 1+\kappa)-\zeta(s, 1+\kappa+i t / \hbar \beta)-\zeta(s, 1+\kappa-i t / \hbar \beta)$. The function $\zeta(z, q)=\sum_{n=0}^{\infty} 1 /(q+n)^{z}$ is the Hurwitz zeta function, related to the Riemann zeta function by $\zeta(z, 1) \equiv \zeta(z)$.

As a further approximation, in addition to the DVR, we restrict the sum over paths of the reduced density matrix in the propagator of equation (12) to the leading contributions. These are given by the class of paths consisting in sojourns in diagonal states interrupted by single off-diagonal excursions called blips. In figure 5 is shown an example of path belonging to this class.

Finally, in the dissipation regimes of intermediate to high temperature, on the scale fixed by $\hbar \omega_{0}$, considered here, the time nonlocal interactions among different blips in equation (13), the inter-blip interactions, can be neglected. This corresponds to a multilevel version [29, 45] of the noninteracting blip approximation (NIBA) [3, 11]. However, the relevant part of the interactions, the intra-blip interactions indicated by the red wavy lines in figure 5, are retained to all orders in the coupling strength.

The NIBA is justified when, on a time scale comparable to the the average interblip time distance, $Q^{\prime}$ assumes its linear form with respect to time and $Q^{\prime \prime}$ becomes approximatively constant [45]. For intrawell blips this time is $\sim \omega_{0}^{-1}$ while for tunneling bilps it is $\sim \Omega_{1,2}^{-1} \gg \omega_{0}^{-1}$. For $s \gtrsim 1$ the linearized form is reached on a time scale that is shorter at higher temperature [3]. With the parameters considered in this work, this time scale is that of the intrawell blips $\sim \omega_{0}^{-1}$ (see figure 6 ). For $s<1$ the interblip interactions are suppressed because, as $Q^{\prime}$ grows rapidly, the exponential cutoff due to the real part of the Feynman-Vernon influence functional becomes severe on the same time scale. Therefore, the NIBA is justified from the sub-Ohmic to the super-Ohmic regime for the intrawell motion. In turn this means that the same approximation scheme is valid $a$ fortiori for the tunneling blips, as their time scale is longer and their effective damping is larger due to the larger distance of DVR states separated by the potential barrier. The above reasoning justifies our generalized-NIBA treatment.

Within the above approximations a generalized master equation (GME) for the populations of the DVR states is derived starting from the explicit path integral expression 


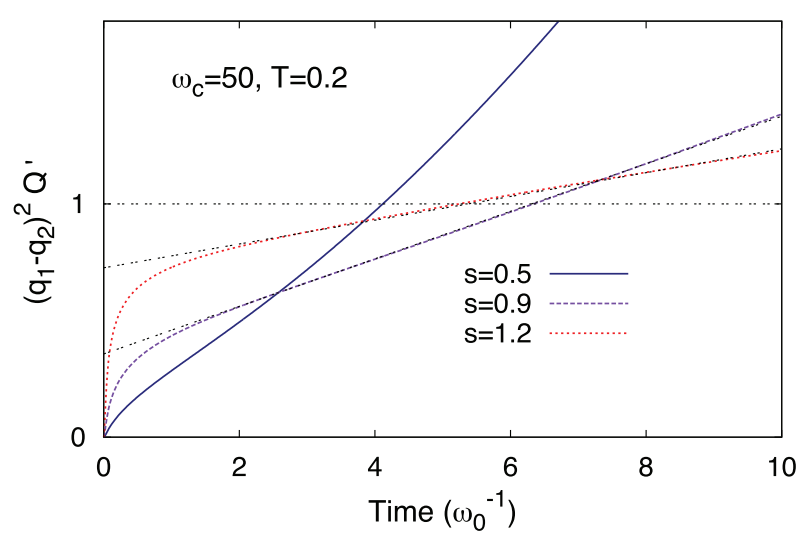

Figure 6. Real part of the pair interaction $Q$ (equation (14)) multiplied by the squared intrawell distance of the left well vs time. For $s \gtrsim 1$ the pair interaction assumes a linearized form on the time scales of the intrawell blips $\sim \omega_{0}^{-1}$. For $s<1$ $Q^{\prime}$ grows rapidly so that the suppression of the interactions among different blips, operated by the real part of the influence phase (equation (13)), is effective on the same time scale. Temperature and cutoff frequency are in units of $\hbar \omega_{0} / k_{\mathrm{B}}$ and $\omega_{0}$, respectively.

for the populations $\rho_{i i}$ at time $t$, given by the propagator in equation (12). The quantum mechanical amplitudes of the paths of the reduced density matrix factorize by virtue of the generalized NIBA, so that the nested time integrals defining the propagator acquire the form of convolutions. As a consequence, the path integral expression for a population in Laplace space takes the form of a series in the number of transitions. The series is resummed and the result transformed back to the time domain, which gives the following GME [45]

$$
\dot{\rho}_{i i}(t)=\sum_{j=1}^{4} \int_{t_{0}}^{t} \mathrm{~d} t^{\prime} K_{i j}\left(t-t^{\prime}\right) \rho_{j j}\left(t^{\prime}\right)+I_{i}(t) .
$$

Aside from the inhomogeneity term, which contains the coherences (off-diagonal elements of the reduced density matrix) at initial time $t_{0}$ and vanishes at long times due to an exponential cutoff, only the populations are involved in the GME. The reason for this is that we start from the formal solution for the populations at final time $t$, and from that solution we derive the GME which relates the populations at time $t$ to their values at earlier times through the kernels $K_{i j}$. The NIBA kernels, which couple the populations to each other in equation (15), are given by

$$
\begin{aligned}
& K_{i j}(t)=2 \Delta_{i j}^{2} \mathrm{e}^{-\left(q_{i}-q_{j}\right)^{2} Q^{\prime}(t)} \cos \left[\epsilon_{i j} t+\left(q_{i}-q_{j}\right)^{2} Q^{\prime \prime}(t)\right] \quad(i \neq j) \\
& K_{j j}(t)=-\sum_{l(\neq j)=1}^{4} K_{l j}(t),
\end{aligned}
$$

where $\epsilon_{i j}=\left(\left\langle q_{i}\left|\hat{H}_{S}\right| q_{i}\right\rangle-\left\langle q_{j}\left|\hat{H}_{S}\right| q_{j}\right\rangle\right) / \hbar$ is the generalization of the bias appearing in the TLS Hamiltonian in the left/right state representation. Note that, due to the prefactors $\left(q_{i}-q_{j}\right)^{2}$ multiplying $Q$, the effective damping strength is much larger for transitions between states in different wells. Moreover, the frequency scales $\Delta_{i j}$ associated to these 
transitions are smaller than those associated to the intrawell transitions. As a consequence, by increasing the coupling strength $\gamma_{s}$, the tunneling oscillations are damped out already at relatively small values of the coupling, while the intrawell oscillations survive until much larger values are reached.

The dynamical regime resulting from our choice of parameters is the crossover regime of damped intrawell oscillations and incoherent tunneling, a regime which lies between the completely coherent and the fully incoherent dynamics. In [46], by using a beyond-NIBA scheme we have investigated this crossover dynamical regime in the Ohmic case down to temperatures for which NIBA-like approximations break down.

Since the relaxation dynamics is governed by the incoherent tunneling and, as shown in figure 7 , the intrawell oscillations are damped out on relatively short time scales, a good estimate for the relaxation time, the time scale on which the system relaxes, is given by the following Markov-approximated version of equation $(15)$ [30, 45]

$$
\dot{\rho}_{i i}(t)=\sum_{j=1}^{4} \Gamma_{i j} \rho_{j j}(t),
$$

where $\Gamma_{i j}=\int_{0}^{\infty} \mathrm{d} t K_{i j}(t)$, with $K_{i j}$ given in equation (16) and where the inhomogeneity term appearing in equation (15) has been neglected. The solution for the population $\rho_{i i}$ is of the form $\rho_{i i}(t)=\rho_{i i}(\infty)+\sum_{k=1}^{3} c_{i k} \exp \left(-\Lambda_{k} t\right)$. The smallest of the rates $\Lambda_{k}$ gives the relaxation time, defined as $\Lambda_{\text {min }}^{-1}$. Notice that in this definition of relaxation time there is no reference to the initial condition. Equation (17) does not capture transient oscillations and is accurate only in the fully incoherent regime. Nevertheless it gives a good estimate for the relaxation time also in the crossover dynamical regime [46]. The master equation (17) has been used to obtain the dynamics and stationary populations in the presence of an external driving [47] and to address the problem of the escape from a quantum metastable state, starting from a nonequilibrium initial condition, with a strongly asymmetric bistable potential and Ohmic dissipation [48].

\section{Dynamics and relaxation times}

In this section we show the results for the dynamics obtained by numerical integration of the GME (equation (15)) with the NIBA kernels given by equation (16) and initial time set to $t_{0}=0$. The numerical scheme used is described in appendix A. Calculations are performed by varying $s$, the exponent of $\omega$ in the spectral density function $J(\omega)$, in the range $0.5 \leqslant s \leqslant 1.2$, and for different cutoff frequencies $\omega_{c}$ (see equation (4)). We consider the two temperatures $T=0.2,0.5 \hbar \omega_{0} / k_{\mathrm{B}}$ and fix the coupling strength to the value $\gamma_{s}=0.1 \omega_{0}$. Throughout this section the system is assumed to be initially in the localized state $\left|q_{1}\right\rangle$ belonging to the left well (see figure 4).

In figure 7 the time evolution of the population $\rho_{11}$ of the state $\left|q_{1}\right\rangle$ is shown at two temperatures and for two values of the cutoff frequency $\omega_{c}$. The time evolutions of $\rho_{11}$ display damped intrawell oscillations ending up in a metastable intrawell equilibrium state which relaxes further towards a stationary configuration over a much larger time scale. The presence of these two different time scales reflects the two different frequency scales 

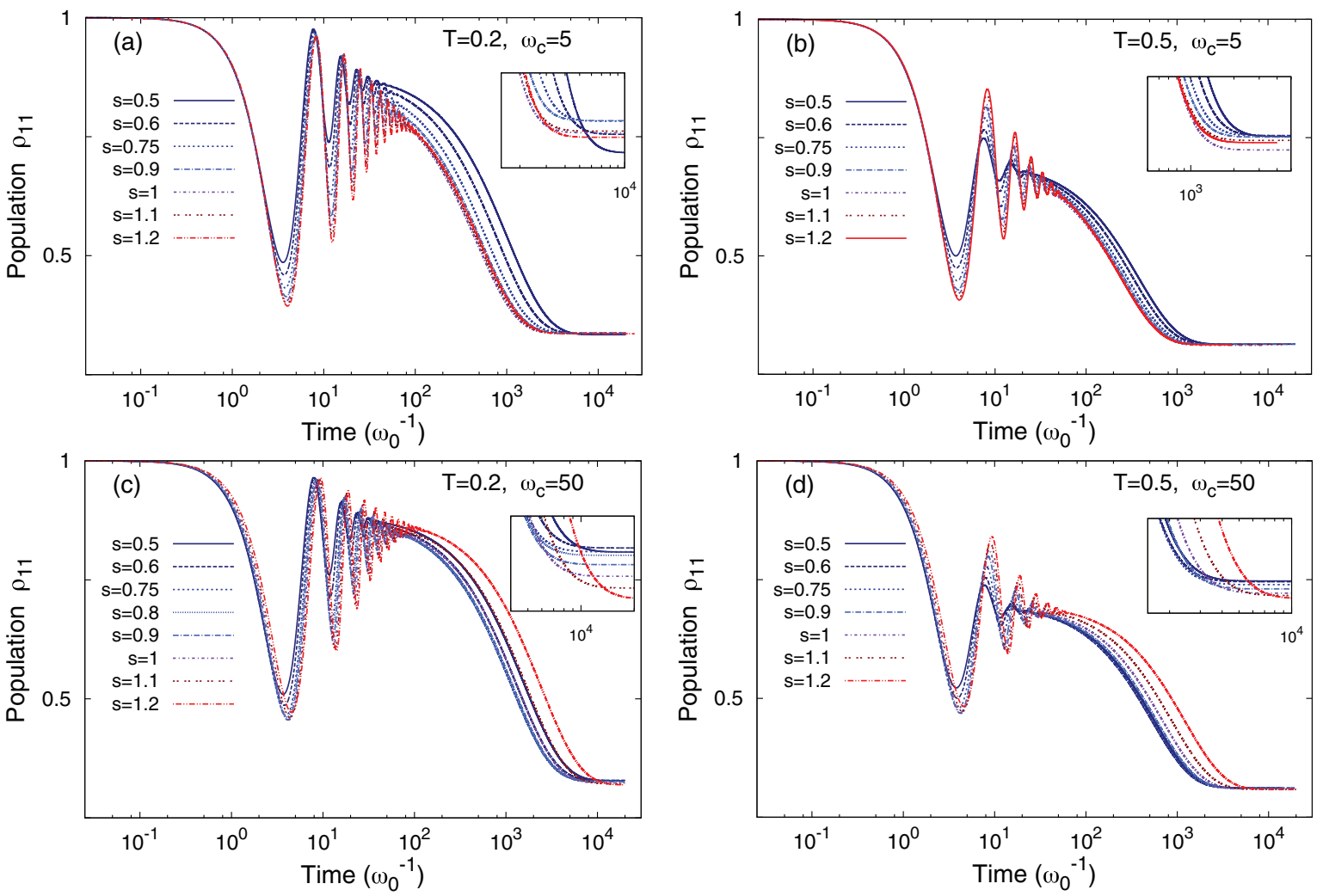

Figure 7. Time evolution of the population $\rho_{11}$ of the state $\left|q_{1}\right\rangle$ for different values of $s$, at two cutoff frequencies $\omega_{c}=5$ (upper panels) and $\omega_{c}=50$ (lower panels) and for two temperatures $T=0.2$ (left panels) and $T=0.5$ (right panels). Insets-Relaxation of $\rho_{11}$ towards its stationary values. The coupling strength $\gamma_{s}$ is fixed to the value $0.1 \omega_{0}$. Temperatures and frequencies are in units of $\hbar \omega_{0} / k_{\mathrm{B}}$ and $\omega_{0}$, respectively.

of tunneling and intrawell motion in the bare system. Moreover the tunneling dynamics is strongly damped due to the distance between states in different wells. This is because the prefactor $\left(q_{i}-q_{j}\right)^{2}$ multiplying $\gamma_{s}$ in the exponent of the NIBA kernels (equation (16)) yields a large effective coupling ( $\gamma_{s}$ is in the prefactor of the function $Q(t)$ defined in equation (14)). We also observe that, in each panel of figure 7, the intrawell oscillations are slower for higher $s$. The effect is more evident at the higher value of the cutoff frequency (compare upper and lower panels for the same temperature). This can be ascribed to a larger renormalized mass (see equation (5)) due to the stronger presence of high frequency modes, especially for the higher cutoff frequency, as exemplified by figure 2. Further, for both cutoff frequencies, the higher is $s$ the less the oscillations are damped. This is because, on the time scale of the intrawell motion, the bath modes contributing to the quantum friction are those with $\omega \lesssim \Omega$, which are denser at lower $s$ (see figure 2). These effects are better seen in figure 8 where the transient dynamics of the population $\rho_{11}$ is shown, with time in linear scale, for the same sets of parameters of panels (a) and (c) of figure 7.

Note also that, by varying $s$, the long time dynamics of $\rho_{11}$ has different behaviors for the two cutoff frequencies. In particular, for $\omega_{c}=5 \omega_{0}$ (upper panels of figure 7) the relaxation is faster at high $s$, while for $\omega_{c}=50 \omega_{0}$ (lower panels) is faster at low $s$. This is also shown in the insets of figure 7 along with the asymptotic behavior. For low $\omega_{c}$ (upper panels) the stationary value of $\rho_{11}$ displays a minor nonmonotonicity as 

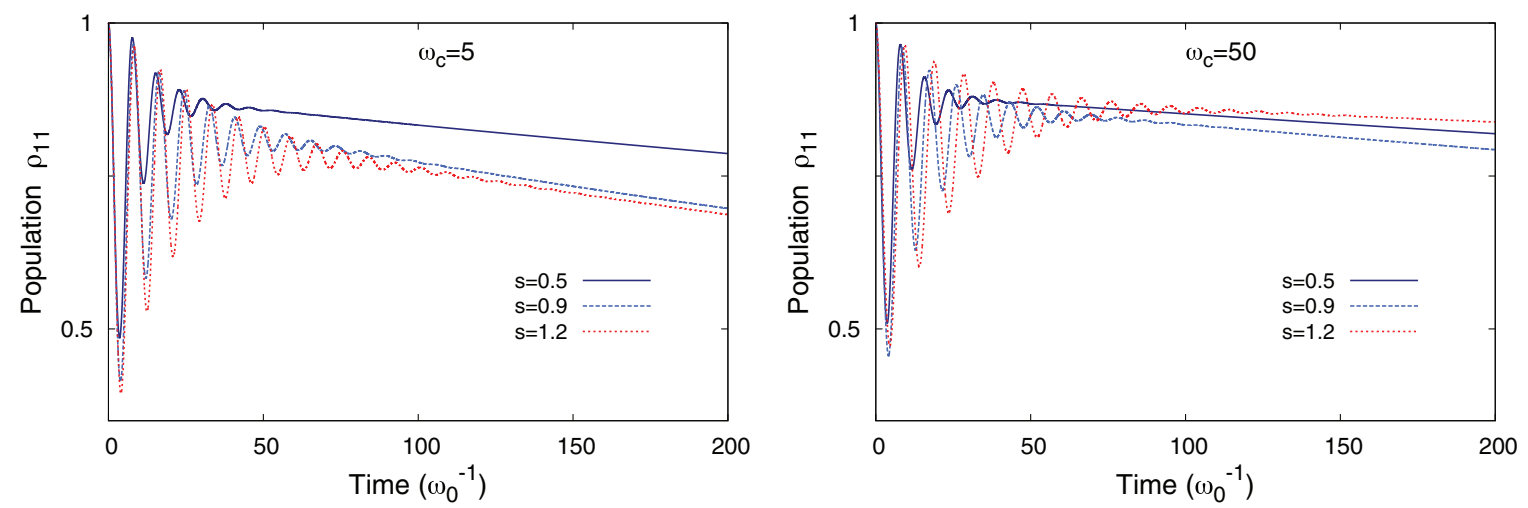

Figure 8. Time evolution of the population $\rho_{11}$ for three values of $s$, at two cutoff frequencies $\omega_{c}=5$ (left panel) and $\omega_{c}=50$ (right panel). Temperature and coupling strength are $T=0.2 \hbar \omega_{0} / k_{\mathrm{B}}$ and $\gamma_{s}=0.1 \omega_{0}$, respectively. $\omega_{c}$ is in units of $\omega_{0}$.

a function of $s$, while for high $\omega_{c}$ (lower panels) decreases as $s$ is increased. The same decrease is found in the stationary value of $\rho_{22}$ (curves not shown). This indicates that, at high $\omega_{c}$, larger values of $s$ determine an increase of the asymptotic population of the lower (right) well (see panels $(e)$ and $(f)$ of figure 9 ).

The features of the relaxation towards the stationary configuration are displayed in figure 9 , where the time evolution of the population difference $P_{\mathrm{L}}-P_{\mathrm{R}}$, where $P_{L(R)}=\rho_{11(33)}+\rho_{22(44)}$, is shown for two temperatures and for three values of the cutoff frequency, along with the relaxation time $\Lambda_{\min }^{-1}$ as a function of $s$ (see section 3.2 for the definition of $\Lambda_{\min }$ ). The $\omega_{c}$-dependent minima in the relaxation time as a function of the exponent $s$, shown in the insets of figure 9 , emerge as the result of two competing mechanisms. On the one hand, by lowering the exponent $s$, the density of low-frequency modes $\left(\omega \sim \Omega_{1,2}\right)$ of the bath is increased (see figure 2 ). These modes act on the time scales of the tunneling $\Omega_{1,2}^{-1}$ contributing to the friction exerted by the heat bath. As a consequence, by moving towards smaller values of $s$, the dissipation is enhanced and the tunneling is hampered. On the other hand, the mass renormalization term, defined by equation (5), increases with $s$ slowing down the relaxation due to the increased inertia of the system. These two competing behaviors yield the minima in the relaxation times. This physical picture is confirmed by the fact that for large $\omega_{c}$, where the mass renormalization effect is stronger, the minimum moves towards lower values of $s$.

To highlight the fact that considering the higher energy doublet yields, in our dissipation regimes, different predictions with respect to a TLS treatment, we calculate the relaxation times versus $s$ for the same physical system within the TLS approximation. The dynamics of $P_{\mathrm{L}}$, the population of the left well state, in the incoherent regime is given by the TLS version of equation (17), which has solution $P_{\mathrm{L}}(t)=\left(\Gamma_{\mathrm{LR}}+\Gamma_{\mathrm{RL}} \mathrm{e}^{-\Gamma t}\right) / \Gamma$, where $\Gamma=\Gamma_{\mathrm{LR}}+\Gamma_{\mathrm{RL}}$. The relaxation time is the inverse of the rate $\Gamma$.

The results are shown in the lower panels of figure 10. The relaxation times are almost two orders of magnitude larger in the TLS approximation. This is because the system has a single frequency scale, namely $\Omega_{1}$, and, with respect to this scale, the coupling $\gamma_{s}=0.1 \omega_{0}$ is very strong. Further, the space separation between left state and right state, which coincides with the distance between the potential minima, is large. On the other hand, in the predictions for the DDS, the presence of the higher energy doublet 

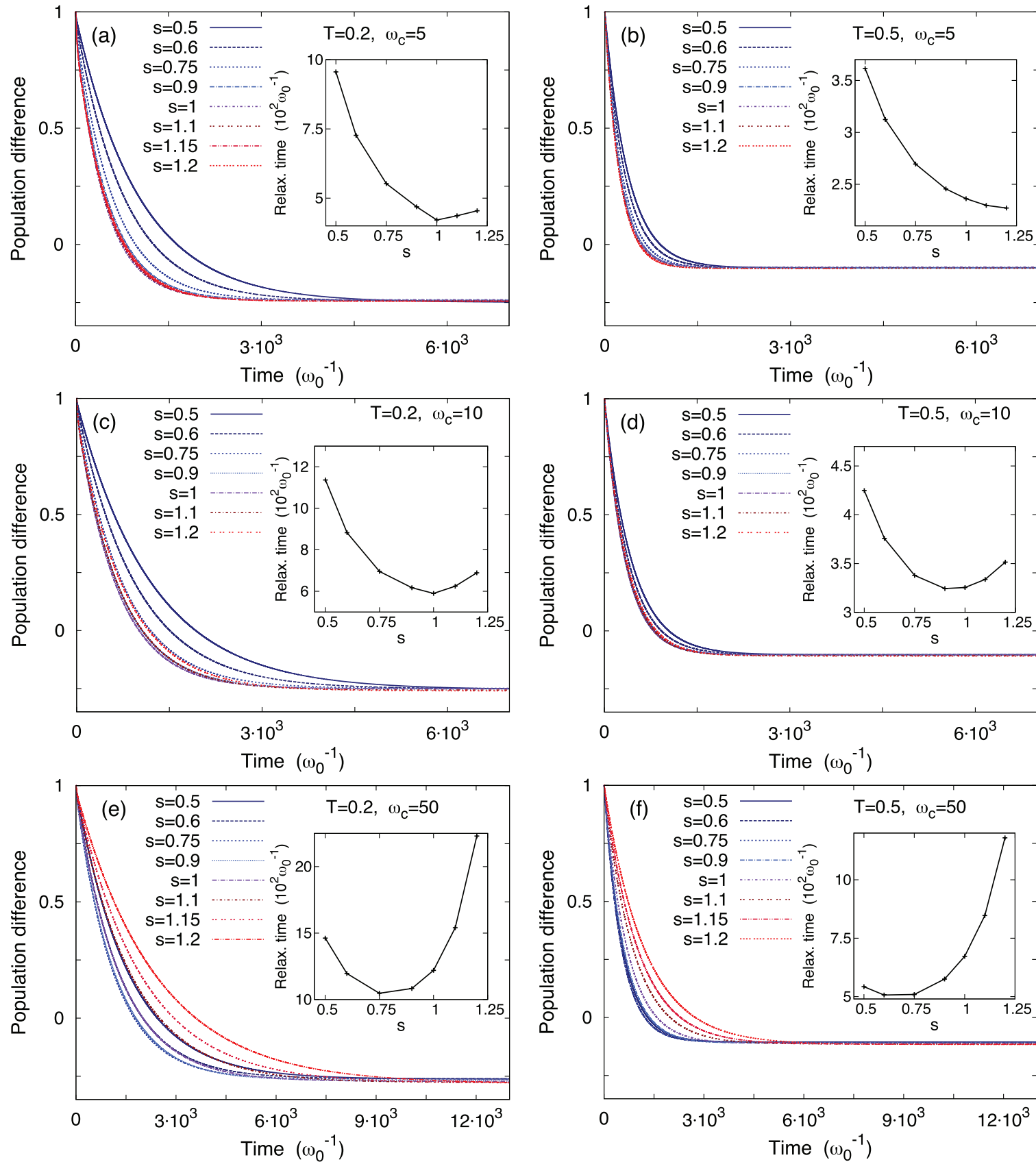

Figure 9. Time evolution of the population difference $P_{\mathrm{L}}-P_{\mathrm{R}}$ for different spectral densities $(0.5 \leqslant s \leqslant 1.2$ ) at temperatures $T=0.2$ (left panels) and $T=0.5$ (right panels) and at cutoff frequencies $\omega_{c}=5$ (upper panels), $\omega_{c}=50$ (central panels), and $\omega_{c}=50$ (lower panels). Insets: relaxation times $\Lambda_{\min }^{-1}$ as a function of $s$. The coupling strength is $\gamma_{s}=0.1 \omega_{0}$. Temperatures and frequencies are in units of $\hbar \omega_{0} / k_{\mathrm{B}}$ and $\omega_{0}$, respectively.

entails the appearance of a second pair of DVR states $\left(\left|q_{2}\right\rangle\right.$ and $\left|q_{3}\right\rangle$, see figure 1) which lie closer to each other and allow for less damped tunneling transitions, shortening the relaxation time (upper panels of figure 10). Nevertheless, the TLS case also shows a minimum. Notice that, at fixed $s$, the prediction for the TLS is that the relaxation time is not very sensitive to the increase in temperature from 0.2 to $0.5 \hbar \omega_{0} / k_{\mathrm{B}}$. On the contrary, 

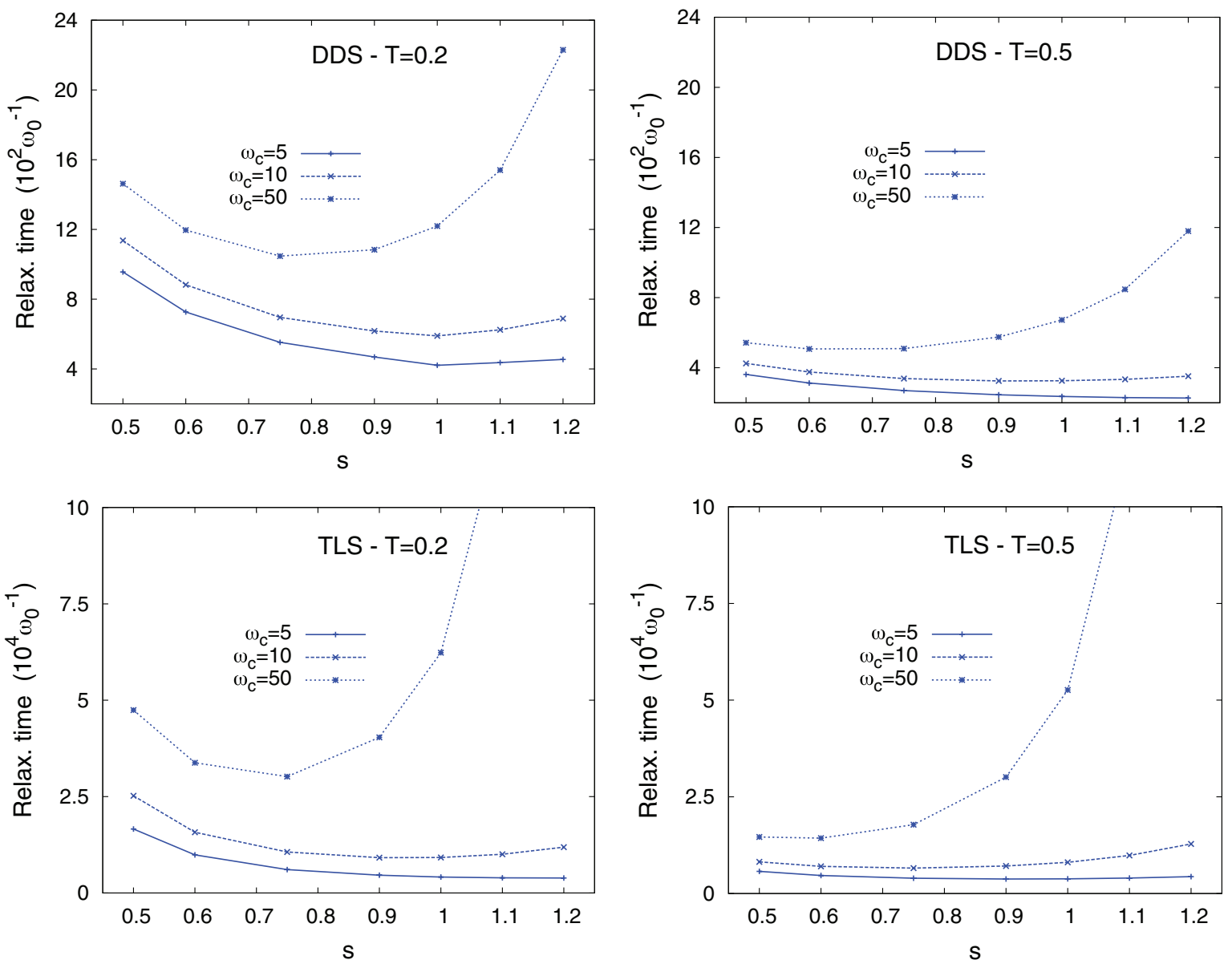

Figure 10. Relaxation time versus $s$. Comparison between the double-doublet system (DDS) approximation (upper panels, same curves as in the insets of figure 9) and the two-level system (TLS) approximation (lower panels). Temperatures and frequencies are in units of $\hbar \omega_{0} / k_{\mathrm{B}}$ and $\omega_{0}$, respectively.

the presence of the higher doublet, which is more excited at higher temperature, causes a speed up of the relaxation dynamics for the four-level system by increasing the temperature. This different dynamical behavior, with respect to an increase of $T$, is in agreement with the analysis made in [10]. There, the presence of an upper energy doublet accounts for the observed enhancement of the tunneling as a function of the temperature.

To conclude this section we spend some words on the role of the initial condition in our analysis. The initial condition chosen, namely $\rho\left(t_{0}\right)=\left|q_{1}\right\rangle\left\langle q_{1}\right|$, involves all of the four energy states through equation (11). Thus the transient dynamics comprises both the relevant time scales of the problem, i.e. the fast intrawell motion and the slow tunneling dynamics, also in the limit of vanishing coupling. In the dissipation regimes considered here this gives rise to the crossover dynamical regime of damped intrawell oscillations and incoherent tunneling shown in the results.

By a suitable combination of DVR states it is possible to construct initial states of our four-level system corresponding to the left and right state $(|L\rangle,|R\rangle)$ involving only the lower energy doublet. If the temperature is low enough, the time evolution of a system prepared in one of these states does not display intrawell oscillations. These involve transitions to higher energy states induced by the heat bath, and a treatment 
based on the TLS approximation is appropriate (see [46] for a detailed exposition). However, at the temperatures considered in our work, especially at $T=0.5 \omega_{0}$, where $\omega_{0}$ sets the order of magnitude of the separation between the first and second energy doublet, intrawell oscillations are expected to be activated regardless of the initial condition. These intrawell oscillations undergo the damping effects described in our analysis. Of course, the transient dynamics for our initial condition is expected to differ from that resulting from a preparation which entails the presence of coherences, with the inhomogeneity term in equation (15) playing a role in the transient. Nevertheless, due to the exponential suppression of this contribution, the relaxation times and the stationary values of the populations are not affected by the initial condition. Based on these considerations, we conclude that, apart from the details of the transient dynamics, the results of our analysis are not modified by initial preparations which determine the presence of the inhomogeneity term in equation (15).

\section{Conclusions}

In this work we investigate the multilevel dissipative dynamics of a quantum bistable system strongly interacting with a heat bath of bosonic modes. The study is carried out by using the Feynman-Vernon influence functional approach for the open system dynamics. The dynamics, in the dissipation regimes considered, is characterized by damped intrawell oscillations and incoherent tunneling. We focus on the influence of the spectral properties of the bosonic heat bath on the transient dynamics. These properties are described by the spectral density function, assumed to be of the form $\omega^{s}$ with a high-frequency cutoff.

By varying the exponent $s$, we find that the intrawell oscillations are less damped and slower at higher $s$ and that the relaxation time has a minimum at a value of $s$ which depends on the cutoff frequency. These effects can be accounted for by considering the interplay of the quantum friction exerted by the low frequency part of the bath and the mass renormalization given by the high frequency modes. By comparing the predictions of our multilevel system with those of the TLS version of the same system we find that, in the regime of temperatures considered, the presence of a higher energy doublet cannot be neglected.

\section{Acknowledgments}

This work was partially supported by MIUR through Grant. No. PON02 _ 00355 3391233, Tecnologie per l'ENERGia e l'Efficienza energETICa-ENERGETIC.

\section{Appendix. Numerical scheme for the generalized master equation}

For the integration of the generalized master equation (15), which is a set of coupled integro-differential equations, we developed a simple implicit numerical scheme that we outline in the following. Although implicit schemes are known to be more stable, 
as compared to explicit ones, they usually require larger computational resources. Specifically, our numerical scheme requires a matrix inversion at each time step. Nevertheless, due to the low dimension of the truncated Hilbert space of the open system considered here and, consequently, of the matrices involved, the integration is carried out with a moderate numerical effort.

The generalized master equation (15), without the inhomogeneity term, reads

$$
\dot{\rho}_{i i}(t)=\sum_{j=1}^{4} \int_{t_{0}}^{t} \mathrm{~d} t^{\prime} K_{i j}\left(t-t^{\prime}\right) \rho_{j j}\left(t^{\prime}\right) .
$$

By using vector notation and setting $t_{0}=0$ the above equation becomes

$$
\dot{\vec{\rho}}(t)=\int_{0}^{t} \mathrm{~d} t^{\prime} \mathbf{K}\left(t-t^{\prime}\right) \vec{\rho}\left(t^{\prime}\right)
$$

where $\mathbf{K}$ is the $4 \times 4$ kernel matrix, with elements $K_{i j}$ defined in equation (16), and $\vec{\rho}=\left(\rho_{11}, \ldots, \rho_{44}\right)$ is the 4 -dimensional population vector. We use the forward finite difference for the derivative and the trapezoid rule for the integral in the RHS. By substituting the time arguments with discrete indexes, e.g. $\vec{\rho}_{m} \equiv \vec{\rho}\left(t_{m}\right)=\vec{\rho}(m \Delta t)$, equation (A.2) acquires the discretized form

$$
\frac{1}{\Delta t}\left(\vec{\rho}_{n+1}-\vec{\rho}_{n}\right)=\Delta t \sum_{m=1}^{n} \mathbf{K}_{n+1, m} \vec{\rho}_{m}+\frac{\Delta t}{2}\left[\mathbf{K}_{n+1,0} \vec{\rho}_{0}+\mathbf{K}_{n+1, n+1} \vec{\rho}_{n+1}\right],
$$

where $\mathbf{K}_{n, m} \equiv \mathbf{K}\left(t_{n}-t_{m}\right)$. Solving for $\vec{\rho}_{n+1}$ we find that the populations at time step $n+1$, in terms of their values at previous times, are given by

$$
\vec{\rho}_{n+1}=\mathbf{M}_{n+1}^{-1} \vec{C}_{n}
$$

where

$$
\mathbf{M}_{n+1} \equiv \mathbf{1}-\frac{(\Delta t)^{2}}{2} \mathbf{K}_{n+1, n+1}
$$

and

$$
\vec{C}_{n} \equiv \vec{\rho}_{n}+\frac{(\Delta t)^{2}}{2} \mathbf{K}_{n+1,0} \vec{\rho}_{0}+(\Delta t)^{2} \sum_{m=1}^{n} \mathbf{K}_{n+1, m} \vec{\rho}_{m}
$$

From equation (A.6) we see that at each time step an integration has to be carried out which starts from initial time. In principle this could prevent the propagation of the solution $\vec{\rho}_{n+1}$ up to long times because of the increasing computational time required as $n$ increases. However, due to the exponential cutoff in the kernels (see equation (16)), the kernel matrix has a finite memory time, say of $M$ time steps, so that $\mathbf{K}_{n, m} \sim \mathbf{0}$ for $n-m \geqslant M$. As a consequence, for $n>M$, we set $\vec{C}_{n} \simeq \vec{\rho}_{n}+(\Delta t)^{2} \sum_{m=n-M}^{n} \mathbf{K}_{n+1, m} \vec{\rho}_{m}$ in equation (A.6). Thus, the solution can be propagated up to long times provided that the time span for which the kernels go to zero is not too large. This is the case for the parameters used throughout the present work. 


\section{References}

[1] Caldeira A O and Leggett A J 1983 Ann. Phys., NY 149 374-456

[2] Caldeira A O and Leggett A J 1981 Phys. Rev. Lett. 46 211-4

[3] Weiss U 2012 Quantum Dissipative Systems 4th edn (Singapore: World Scientific)

[4] Spagnolo B, Caldara P, La Cognata A, Valenti D, Fiasconaro A, Dubkov A A and Falci G 2012 Int. J. Mod. Phys. B 261241006

[5] Le Hur K 2008 Ann. Phys., NY 323 2208-40

[6] Wang H and Thoss M 2008 New J. Phys. 10115005

[7] Cukier R I and Morillo M 1990 J. Chem. Phys. 93 2364-9

[8] Gatteschi D, Sessoli R and Villain J 2006 Molecular Nanomagnets (Oxford: Oxford University Press)

[9] Chatterjee B, Brouzos I, Zöllner S and Schmelcher P 2010 Phys. Rev. A 82043619

[10] Johnson M W et al 2011 Nature 473 194-8

[11] Leggett A J, Chakravarty S, Dorsey A T, Fisher M P A, Garg A and Zwerger W 1987 Rev. Mod. Phys. 59 1-85

[12] Egger R and Mak C H 1994 Phys. Rev. B 50 15210-20

[13] Nesi F, Paladino E, Thorwart M and Grifoni M 2007 Phys. Rev. B 76155323

[14] Nalbach P and Thorwart M 2010 Phys. Rev. B 81054308

[15] Nalbach P and Thorwart M 2013 Phys. Rev. B 87014116

[16] Kast D and Ankerhold J 2013 Phys. Rev. Lett. 110010402

[17] Bera S, Florens S, Baranger H U, Roch N, Nazir A and Chin A W 2014 Phys. Rev. B 89121108

[18] Makri N and Makarov D E 1995 J. Chem. Phys. 102 4600-10

[19] McCutcheon D P S, Dattani N S, Gauger E M, Lovett B W and Nazir A 2011 Phys. Rev. B 84081305

[20] Daley A J, Kollath C, Schollwöck U and Vidal G 2004 J. Stat. Mech. P04005

[21] Bulla R, Costi T and Pruschke T 2008 Rev. Mod. Phys. 80 395-450

[22] Prior J, Chin A W, Huelga S F and Plenio M B 2010 Phys. Rev. Lett. 105050404

[23] Egger R, Mühlbacher L and Mak C H 2000 Phys. Rev. E 61 5961-6

[24] Wang H and Thoss M 2003 J. Chem. Phys. 119 1289-99

[25] Stockburger J T and Grabert H 2002 Phys. Rev. Lett. 88170407

[26] Ishizaki A and Tanimura Y 2005 J. Phys. Soc. Japan 74 3131-4

[27] Moix J M and Cao J 2013 J. Chem. Phys. 139134106

[28] Feynman R P and Vernon F L Jr 1963 Ann. Phys., NY 24 118-73

[29] Grifoni M, Sassetti M and Weiss U 1996 Phys. Rev. E 53 R2033-6

[30] Thorwart M, Grifoni M and Hänggi P 2000 Phys. Rev. Lett. 85 860-3

[31] Wilner E Y, Wang H, Thoss M and Rabani E 2015 Phys. Rev. B 92195143

[32] Magazzù L, Valenti D, Carollo A and Spagnolo B 2015 Entropy 17 2341-54

[33] Poletto S, Chiarello F, Castellano M G, Lisenfeld J, Lukashenko A, Cosmelli C, Torrioli G, Carelli P and Ustinov A V 2009 New J. Phys. 11013009

[34] Augello G, Valenti D and Spagnolo B 2010 Eur. Phys. J. B 78 225-34

[35] Valenti D, Guarcello C and Spagnolo B 2014 Phys. Rev. B 89214510

[36] Devoret M H, Martinis J M, Esteve D and Clarke J 1984 Phys. Rev. Lett. 53 1260-3

[37] Devoret M H, Martinis J M and Clarke J 1985 Phys. Rev. Lett. 55 1908-11

[38] Han S, Lapointe J and Lukens J E 1991 Phys. Rev. Lett. 66 810-3

[39] Chiorescu I, Nakamura Y, Harmans C J P M and Mooij J E 2003 Science 299 1869-71

[40] Chiarello F, Paladino E, Castellano M G, Cosmelli C, D’Arrigo A, Torrioli G and Falci G 2012 New J. Phys. 14023031

[41] Gillet J, Garcia-March M A, Busch T and Sols F 2014 Phys. Rev. A 89023614

[42] Grabert H, Schramm P and Ingold G L 1988 Phys. Rep. 168 115-207

[43] Harris D O, Engerholm G G and Gwinn W D 1965 J. Chem. Phys. 43 1515-7

[44] Light J C and Carrington T 2007 Discrete-Variable Representations and their Utilization (Advances in Chemical Physics vol 114) (New York: Wiley) pp 263-310

[45] Thorwart M, Grifoni M and Hänggi P 2001 Ann. Phys., NY 293 15-66

[46] Magazzù L, Valenti D, Spagnolo B and Grifoni M 2015 Phys. Rev. E 92032123

[47] Magazzù L Valenti D, Caldara P, La Cognata A, Spagnolo B and Falci G 2013 Acta Phys. Pol. B 441185

[48] Valenti D, Magazzù L, Caldara P and Spagnolo B 2015 Phys. Rev. B 91235412 